PROCEEDINGS OF THE

AMERICAN MATHEMATICAL SOCIETY

Volume 128, Number 9, Pages 2819-2827

S 0002-9939(00)05340-5

Article electronically published on February 29, 2000

\title{
VON NEUMANN BETTI NUMBERS AND NOVIKOV TYPE INEQUALITIES
}

\author{
MICHAEL FARBER
}

(Communicated by Jozef Dodziuk)

\begin{abstract}
In this paper we show that Novikov type inequalities for closed 1-forms hold with the von Neumann Betti numbers replacing the Novikov numbers. As a consequence we obtain a vanishing theorem for $L^{2}$ cohomology. We also prove that von Neumann Betti numbers coincide with the Novikov numbers for free abelian coverings.
\end{abstract}

\section{$\S 0$. INTRODUCTION}

S. Novikov and M. Shubin [NS proved that Morse inequalities for smooth functions remain true with the usual Betti numbers being replaced by the von Neumann Betti numbers.

Novikov $\mathrm{N}$ initiated an analog of the Morse theory for closed 1-forms. He showed that the Morse inequalities for functions can be generalized to closed 1forms if instead of the Betti numbers one uses the Novikov numbers (they will be briefly reviewed in $\S 3.1$ below).

In this paper we show that the inequalities of Novikov and Shubin [NS] hold for closed 1-forms as well. This result gives new Novikov type inequalities for closed 1-forms. Viewed differently, this gives a vanishing theorem for $L^{2}$ cohomology, generalizing a theorem of W. Lück [L1].

The proof of our Theorem 1 uses an idea of Gromov and Eliashberg [EG], which consists of counting additional critical points which appear when transforming the given closed 1-form into a function; the proof also uses the multiplicativity of the von Neumann Betti numbers under finitely sheeted coverings.

In papers [BF2] and [MS] different Novikov type inequalities using the von Neumann Betti numbers were put forward. These inequalities involve cohomology of flat bundles of Hilbertian modules twisted by a generic line bundle determined by the closed 1-form (similarly to the finite dimensional case). Our approach in this paper does not require the twisting, and therefore it is simpler. On the other hand V. Mathai and M. Shubin [MS] allow more general Hilbertian flat bundles.

It is clear that one may easily generalize Theorem 1 below for closed 1-forms with Bott type singularities, in a fashion similar to [BF1] and [BF2].

I would like to thank Andrew Ranicki for stimulating discussions.

Received by the editors October 19, 1998.

1991 Mathematics Subject Classification. Primary 58Exx; Secondary 57R19.

This research was partially supported by the US - Israel Binational Science Foundation, by the Herman Minkowski Center for Geometry, and by EPSRC grant GR/M20563. 


\section{$\S 1$. The MAIN RESUlts}

First we recall the basic notions.

A closed 1-form $\omega$ locally is a differential of a function $\left.\omega\right|_{U}=d f_{U}$, where $f_{U}$ is a smooth function on $U$, determined up to a constant. Hence, all local properties of functions (e.g. the notions of a critical point, Morse singularities, indices) immediately generalize to closed 1-forms. For example, a point $p \in U$ is a critical point of $\omega$ if $p$ is a critical point of $f_{U}$ (which is equivalent to the requirement that $\omega_{p}=0$ ); $p$ a nondegenerate (or Morse) critical point of the form $\omega$ if it is a Morse critical point of the function $f_{U}$, etc.

1. Theorem. Let $M$ be a closed smooth manifold and let $\omega$ be a closed 1-form on $M$, having only Morse singularities. Let $\pi^{\prime} \subset \pi=\pi_{1}(M)$ be a normal subgroup with the following property: for any loop $\gamma \in \pi^{\prime}, \int_{\gamma} \omega=0$ holds. Then

$$
\sum_{k=0}^{i}(-1)^{k} c_{i-k}(\omega) \geq \sum_{k=0}^{i}(-1)^{k} b_{i-k}^{(2)}\left(\tilde{M} ; \pi / \pi^{\prime}\right), \quad i=0,1,2, \ldots,
$$

where $c_{i}(\omega)$ denotes the number of critical points of $\omega$ having index $i, \tilde{M}$ denotes the covering of $M$ corresponding to $\pi^{\prime}$, and $b_{i}^{(2)}\left(\tilde{M} ; \pi / \pi^{\prime}\right)$ denotes the von Neumann Betti number of $\tilde{M}$. In particular, (1-1) implies

$$
c_{i}(\omega) \geq b_{i}^{(2)}\left(\tilde{M} ; \pi / \pi^{\prime}\right) .
$$

For the definitions of von Neumann Betti numbers and their main properties we refer to $[\mathrm{A}], \mathrm{CG}], \mathrm{L} 2], \mathrm{F} 2]$.

Theorem 1 obviously implies Corollaries 2 and 3, cf. below. These Corollaries generalize a theorem of Wolfgang Lück [L1 (conjectured by M. Gromov), stating that $L^{2}$-Betti numbers of a manifold, fibering over the circle, vanish.

2. Corollary (Vanishing Theorem). Let $M$ be a closed smooth manifold admitting a closed 1-form with Morse type singularities having no critical points of index $j$ for some $0<j<n$. Then for any normal subgroup $\pi^{\prime} \subset \pi$, lying in the commutator subgroups of $\pi=\pi_{1}(M)$, the $j$-dimensional von Neumann Betti number vanishes

$$
b_{j}^{(2)}\left(\tilde{M} ; \pi / \pi^{\prime}\right)=0
$$

3. Corollary (Morse Lacunary Principle). Let $M$ be a closed manifold admitting a closed 1-form $\omega$ with Morse type singularities so that all the critical points of $\omega$ have even indices. Then for any normal subgroup $\pi^{\prime} \subset \pi$, lying in the commutator subgroups of $\pi=\pi_{1}(M)$, the odd-dimensional von Neumann Betti numbers vanish $b_{2 j-1}^{(2)}\left(\tilde{M} ; \pi / \pi^{\prime}\right)=0$ and the even-dimensional von Neumann Betti numbers are given by the formula

$$
b_{2 j}^{(2)}\left(\tilde{M} ; \pi / \pi^{\prime}\right)=c_{2 j}(\omega) .
$$

Note that Theorem 0.3 of [BF1] implies that under the conditions of Corollary 3 the number $c_{2 j}(\omega)$ equals the Novikov number $b_{2 j}(\xi)$, where $\xi \in H^{1}(M ; \mathbf{R})$ is the cohomology class of $\omega$. Hence we obtain the equality

$$
b_{2 j}^{(2)}\left(\tilde{M} ; \pi / \pi^{\prime}\right)=b_{2 j}(\xi)
$$

compare Theorem 5 below. 
Definitions of the Novikov numbers can be found in the literature BF1, F1, and $[\mathrm{N}$. Paper [BF1] contains a stronger version of Novikov type inequalities and a more general notion of Novikov numbers. In this paper we will use only the standard Novikov numbers $b_{i}(\xi)$. It will be convenient for our purposes to be based on the definition given in [BF1, cf. $\S 3.1$.

4. Corollary (Symplectic circle actions). Let $M$ be a closed symplectic manifold admitting a symplectic circle action with isolated fixed points. Then for any normal subgroup $\pi^{\prime} \subset \pi$, lying in the commutator subgroups of $\pi=\pi_{1}(M)$, the odddimensional von Neumann Betti numbers vanish $b_{2 j-1}^{(2)}\left(\tilde{M} ; \pi / \pi^{\prime}\right)=0$, and the evendimensional von Neumann Betti numbers coincide with the Novikov numbers

$$
b_{2 j}^{(2)}\left(\tilde{M} ; \pi / \pi^{\prime}\right)=b_{2 j}(\xi) .
$$

Here $\xi \in H^{1}(M ; \mathbf{R})$ denotes the cohomology class of the generalized moment map, and $b_{i}(\xi)$ denotes the $i$-dimensional Novikov number corresponding to $\xi$.

Proof of Corollary 4. Let us first explain the terms used in Corollary 4. Suppose that $\Omega$ denotes the symplectic form of $M$. The $S^{1}$ action is assumed to be symplectic, which means that, for any $g \in S^{1}, g^{*} \Omega=\Omega$ holds. Let $X$ denote the vector field generating the $S^{1}$-action. Then

$$
\omega=\iota(X) \Omega
$$

is a closed 1-form on $M$, which is called the generalized moment map. We consider its De Rham cohomology class $\xi=[\omega] \in H^{1}(M ; \mathbf{R})$. Recall that a symplectic circle action is called Hamiltonian if $\xi=0$. In this case the Novikov numbers $b_{i}(\xi)$ coincide with the Betti numbers $b_{i}(M)$.

The critical points of the generalized moment map $\omega$ are precisely the fixed points of the circle action. It is well known that $\omega$ has Morse type singularities, assuming that the fixed points are isolated; moreover, all the critical points have even indices; cf. $\mathrm{Au}$. Hence from Corollary 3 we obtain that $b_{2 j-1}^{(2)}\left(\tilde{M} ; \pi / \pi^{\prime}\right)=0$ and the even-dimensional von Neumann Betti numbers are given by the formula (1-4). Now we will use the main theorem 0.3 of [BF1, which gives

$$
c_{2 j}(\omega)=b_{2 j}(\xi)
$$

The following theorem states that in the special case when $\pi^{\prime}$ coincides with the $\operatorname{ker}(\xi)$, the inequalities of Theorem 1 are precisely the Novikov inequalities.

5. Theorem (Novikov numbers equal $L^{2}$ Betti numbers). Let $M$ be a closed smooth manifold and let $\xi \in H^{1}(M ; \mathbf{R})$ be a nontrivial cohomology class. Let $\pi^{\prime}$ be the kernel of the homomorphism $\xi: \pi=\pi_{1}(M) \rightarrow \mathbf{R}$ determined by $\xi$. Then the $i$-dimensional Novikov number $b_{i}(\xi)$ coincides with the $i$-dimensional von Neumann Betti number of the covering $\tilde{M} \rightarrow M$ corresponding to $\pi^{\prime}$ :

$$
b_{i}(\xi)=b_{i}^{(2)}\left(\tilde{M} ; \pi / \pi^{\prime}\right) .
$$

The plan of the paper is as follows. The proof of Theorem 1 is presented in $\S 2$. The proof of Theorem 5 is described in $\S 3$. 


\section{§2. Proof of Theorem 1}

Let $\omega$ be a closed 1-form on a closed manifold $M$. First, we will assume that the form $\omega$ has integral periods, i.e. its cohomology class is integral $\xi=[\omega] \in H^{1}(M ; \mathbf{Z})$; the general case will be treated at the end of the proof.

There exists a smooth map into the circle $f: M \rightarrow S^{1}$ with $\omega=f^{*}(d \theta)$, where $d \theta$ denotes the standard angular form on the circle. This map is given by

$$
f(x)=\exp \left(2 \pi i \int_{x_{0}}^{x} \omega\right)
$$

where $x_{0} \in M$ is a base point. We will also denote by $\xi: \pi=\pi_{1}(M) \rightarrow \mathbf{Z}$ the induced homomorphism $[\gamma] \mapsto \int_{\gamma} \omega$, where $\gamma$ is a loop in $M$.

Given a positive integer $m$, we will denote by $\pi_{m}$ the preimage $\pi_{m}=\xi^{-1}(m \mathbf{Z})$. It is a normal subgroup containing $\pi^{\prime}$. Denote by $\tilde{M}_{m}$ the cyclic $m$-sheeted covering $\tilde{M}_{m} \rightarrow M$ corresponding to the subgroup $\pi_{m}$. It is clear that the pullback $\tilde{\omega}_{m}$ of the form $\omega$ to $\tilde{M}_{m}$ has the following Morse numbers:

$$
c_{j}\left(\tilde{\omega}_{m}\right)=m \cdot c_{j}(\omega) .
$$

Claim. There exists a constant $C$, independent of $m$, so that on each manifold $\tilde{M}_{m}$ there exists an exact 1-form $\tilde{\omega}_{m}^{\prime}$ with

$$
c_{j}\left(\tilde{\omega}_{m}\right) \leq c_{j}\left(\tilde{\omega}_{m}^{\prime}\right) \leq c_{j}\left(\tilde{\omega}_{m}\right)+C, \quad j=0,1, \ldots
$$

We will postpone the proof of the Claim and will continue the proof of the Theorem.

We have the inequality

$$
\begin{aligned}
& \sum_{k=0}^{i}(-1)^{k} c_{i-k}\left(\tilde{\omega}_{m}^{\prime}\right) \\
& \quad \geq \sum_{k=0}^{i}(-1)^{k} b_{i-k}^{(2)}\left(\tilde{M} ; \pi_{m} / \pi^{\prime}\right), \quad i=0,1,2, \ldots
\end{aligned}
$$

which is just the Novikov - Shubin inequality [NS] (cf. also [MS]) applied to the exact form $\tilde{\omega}_{m}^{\prime}$ on the compact manifold $\tilde{M}_{m}$.

Multiplicativity of the von Neumann Betti numbers under finitely sheeted coverings gives

$$
b_{j}^{(2)}\left(\tilde{M} ; \pi_{m} / \pi^{\prime}\right)=m \cdot b_{j}^{(2)}\left(\tilde{M} ; \pi / \pi^{\prime}\right)
$$

cf. [L2], Theorem 1.7, statement 7 . Here we use our assumption that $\pi^{\prime} \subset \operatorname{ker}(\xi)$. 
Combining (2-1), (2-2), (2-3), (2-4) we will have

$$
\begin{aligned}
\sum_{k=0}^{i}( & -1)^{k} c_{i-k}(\omega) \\
& =\frac{1}{m} \sum_{k=0}^{i}(-1)^{k} c_{i-k}\left(\tilde{\omega}_{m}\right) \\
& \geq \frac{1}{m}\left[\sum_{k=0}^{i}(-1)^{k} c_{i-k}\left(\tilde{\omega}_{m}^{\prime}\right)-(i+1) \cdot C\right] \\
& \geq \frac{1}{m}\left[\sum_{k=0}^{i}(-1)^{k} b_{i-k}^{(2)}\left(\tilde{M} ; \pi_{m} / \pi^{\prime}\right)-(i+1) \cdot C\right] \\
& =\sum_{k=0}^{i}(-1)^{k} b_{i-k}^{(2)}\left(\tilde{M} ; \pi / \pi^{\prime}\right)-\frac{(i+1) \cdot C}{m} .
\end{aligned}
$$

Hence,

$$
\sum_{k=0}^{i}(-1)^{k} c_{i-k}(\omega) \geq \sum_{k=0}^{i}(-1)^{k} b_{i-k}^{(2)}\left(\tilde{M} ; \pi / \pi^{\prime}\right)-\frac{(i+1) \cdot C}{m}
$$

for arbitrary $m>0$. Since $C$ is independent of $m$, taking the limit $m \rightarrow \infty$ in the obtained inequality (2-6) proves (1-1).

Now we want to prove the Claim.

Suppose that $\exp \left(2 \pi i \theta_{0}\right) \in S^{1}$ is a regular value of the map $f: M \rightarrow S^{1}$, where $0<\theta_{0}<1$. Let $V \subset M$ denote $f^{-1}\left(\theta_{0}\right)$. Consider the cylinder $V \times[-1,1]$ and find an arbitrary Morse function

$$
\phi: V \times[-1,1] \rightarrow \mathbf{R}
$$

with the following properties:

(a) $\phi$ assumes values in the open interval $(0,1)$;

(b) $\phi(v, t)=1+\delta t$ for all $t \in[-1,-1 / 2)$ and $v \in V$, where $0<\delta<1$ is a fixed number;

(c) $\phi(v, t)=\delta t$ for all $t \in(1 / 2,1]$ and $v \in V$.

We will denote by $C$ the total number of critical points of $\phi$.

For any $m$ we have the following commutative diagram:

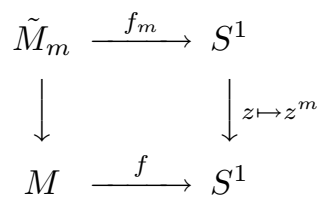

We obtain $\tilde{\omega}_{m}=f_{m}^{*}(d \theta)$, where $f_{m}: \tilde{M}_{m} \rightarrow S^{1}$ is a smooth map. It is clear that for any $m$ we may smoothly imbed $V \times[-1,1]$ into $\tilde{M}_{m}$ so that on the image of $V \times[-1,1]$, the map $f_{m}$ is given by $f_{m}(v, t)=\exp \left(2 \pi i\left[\theta_{0} / m+\delta t\right]\right)$. We will now construct a new map $g_{m}: \tilde{M}_{m} \rightarrow S^{1}$ as follows:

(a) $g_{m}$ coincides with $f_{m}$ on the complement of $V \times[-1,1]$ in $\tilde{M}_{m}$;

(b) on $V \times[-1,1]$ the map $g_{m}$ is given by

$$
g_{m}(v, t)=\exp \left(2 \pi i\left[\theta_{0} / m+\phi(v, t)\right]\right),
$$

where $\phi: V \times[-1,1] \rightarrow \mathbf{R}$ is the function constructed above. 
Set $\tilde{\omega}_{m}^{\prime}=g_{m}^{*}(d \theta)$. Then the obtained 1-form $\tilde{\omega}_{m}^{\prime}$ is exact, since the map $g_{m}$ : $\tilde{M}_{m} \rightarrow S^{1}$ is null homotopic (it does not assume the value $\left.\exp \left(2 \pi i \theta_{0} / m\right) \in S^{1}\right)$. The inequality (2-2) is clearly satisfied, since the forms $\tilde{\omega}_{m}$ and $\tilde{\omega}_{m}^{\prime}$ have the same critical points on the complement of $V \times[-1,1]$ and on $V \times[-1,1]$ the form $\tilde{\omega}_{m}$ has no critical points, while the form $\tilde{\omega}_{m}^{\prime}$ may have at most $C$ critical points.

This proves the Claim and hence completes the proof of Theorem 1 in the case of closed 1-forms $\omega$ having integral cohomology classes.

Consider now a closed 1-form $\omega$ representing an arbitrary cohomology class $\xi=[\omega] \in H^{1}(M ; \mathbf{R})$ and having Morse type singularities. It clearly defines a homomorphism $\xi: H_{1}(M ; \mathbf{Z}) \rightarrow \mathbf{R}$, and the image $\operatorname{im}(\xi)$ is a finitely generated free abelian group. We will denote the rank of this image by $r=\operatorname{rank}(\operatorname{im}(\xi))=\operatorname{rank}(\omega)$.

Note that any closed 1-form $\omega$ having rank 1 can be represented as $\omega=\lambda \omega_{0}$, where $\lambda>0$ and $\omega_{0}$ represents an integral class. Since the forms $\omega$ and $\omega_{0}$ have the same critical points, we conclude that Theorem 1 holds for all closed 1-forms of rank 1.

Suppose now that $\omega$ is a closed 1-form having rank $r>1$. Then we may find a sequence of closed 1-forms $\omega_{n}$ on $M$ with the following properties:

(i) $\omega_{n}$ converges to $\omega$ in the $C^{0}$-norm;

(ii) there exists a neighbourhood $U$ of the set $S$ of critical points of $\omega$ such that $\left.\omega\right|_{U}=\left.\omega_{n}\right|_{U}$ for all $n$;

(iii) $\omega_{n}$ has rank 1 for every $n$;

(iv) the homomorphism $\left[\omega_{n}\right]: H_{1}(M ; \mathbf{Z}) \rightarrow \mathbf{R}$ vanishes on $\operatorname{ker}(\xi)$.

Because of (i) and (ii), for large $n$ the form $\omega_{n}$ has the same critical points as $\omega$ and they have the same indices $c_{j}(\omega)=c_{j}\left(\omega_{n}\right)$. Because of properties (iii) and (iv) we have the inequality

$$
\sum_{k=0}^{i}(-1)^{k} c_{i-k}\left(\omega_{n}\right) \geq \sum_{k=0}^{i}(-1)^{k} b_{i-k}^{(2)}\left(\tilde{M} ; \pi / \pi^{\prime}\right), \quad i=0,1,2, \ldots,
$$

which obviously implies (1-1).

Let us show how to construct the sequence of forms $\omega_{n}$. Let $N_{\xi} \subset H^{1}(M ; \mathbf{R})$ be the subspace formed by classes $\eta$ with $\left.\eta\right|_{\operatorname{ker}(\xi)}=0$; here $r=\operatorname{rank}(\xi)$. Let $\eta_{1}, \ldots, \eta_{r}$ be a basis of $N_{\xi}$ such that $\operatorname{rank}\left(\eta_{j}\right)=1$ for $j=1, \ldots, r$. We may realize each class $\eta_{j}$ by a closed 1-form $\nu_{j}$ which vanishes identically in a neighbourhood of the zeros of $\omega$. We may write $\xi=\sum_{j=1}^{r} \alpha_{j} \eta_{j}$, where $\alpha_{j} \in \mathbf{R}$. Now, choose a sequence of rational numbers $\alpha_{j}^{n}$ converging to $\alpha_{j}$ as $n \rightarrow \infty$. Then the sequence of closed 1 -forms

$$
\omega_{n}=\omega+\sum_{j=1}^{r}\left(\alpha_{j}^{n}-\alpha_{j}\right) \nu_{j}
$$

satisfies all the requirements. This completes the proof.

\section{§3. Proof of Theorem 5}

The proof of Theorem 5 will use basic harmonic analysis.

Before starting the proof we recall the definition of the Novikov numbers.

3.1. The Novikov numbers $b_{i}(\xi)$. Let $\xi \in H^{1}(M ; \mathbf{R})$ be a cohomology class. We will denote by $\operatorname{ker}(\xi)$ the kernel of the induced homomorphism $\xi: H_{1}(M ; \mathbf{Z}) \rightarrow \mathbf{R}$.

Let us consider complex flat line bundles $L \rightarrow M$ with the following property: the monodromy along any loop $\gamma \in \operatorname{ker}(\xi)$ is identity. We will denote by $\mathcal{V}_{\xi}$ the 
set of isomorphism classes of all such flat line bundles. Given $L \in \mathcal{V}_{\xi}$, it has the monodromy homomorphism

$$
\operatorname{Mon}_{\mathrm{L}}: H_{1}(M ; \mathbf{Z}) / \operatorname{ker}(\xi) \rightarrow \mathbf{C}^{*},
$$

which completely determines the isomorphism type of $L$. Hence, there is a one-toone correspondence between $\mathcal{V}_{\xi}$ and the complex torus $\left(\mathbf{C}^{*}\right)^{r}$. Here $r$ is the rank of class $\xi$, i.e. $r=\operatorname{rank}\left(H_{1}(M ; \mathbf{Z}) / \operatorname{ker}(\xi)\right)=\operatorname{rank}(\xi)$. This identification $\mathcal{V}_{\xi} \simeq\left(\mathbf{C}^{*}\right)^{r}$ allows us to view $\mathcal{V}_{\xi}$ as an affine algebraic variety.

Fix some $i$ (with $0 \leq i \leq \operatorname{dim} M$ ) and consider the function

$$
\mathcal{V}_{\xi} \ni L \mapsto \operatorname{dim}_{\mathbf{C}} H_{i}(M ; L) .
$$

It is well known in algebraic geometry (cf. $[\mathrm{H}]$, chapter $3, \S 12)$ that function $(3-1)$ has the following property: there exists a proper algebraic subvariety $V=V_{i}(M) \subset$ $\mathcal{V}_{\xi}$ such that the dimension $\operatorname{dim}_{\mathbf{C}} H_{i}(M ; L)$ is constant for all $L \notin V$ and for $L^{\prime} \in V$

$$
\operatorname{dim}_{\mathbf{C}} H_{i}\left(M ; L^{\prime}\right)>\operatorname{dim}_{\mathbf{C}} H_{i}(M ; L) .
$$

Definition. The Novikov number $b_{i}(\xi)$ is defined as $\operatorname{dim}_{\mathbf{C}} H_{i}(M ; L)$ for $L \in \mathcal{V}_{\xi}-V$.

3.2. Unitary flat bundles. Consider the subset $\mathcal{T}_{\xi} \subset \mathcal{V}_{\xi}$ consisting of isomorphism classes of flat line bundles $L$ admitting flat Hermitian metrics. Under the identification $\mathcal{V}_{\xi} \simeq\left(\mathbf{C}^{*}\right)^{r}$ given by the monodromy representation, the subset $\mathcal{T}_{\xi}$ corresponds to the real torus $\left(S^{1}\right)^{r} \subset\left(\mathbf{C}^{*}\right)^{r}$. This shows, in particular, that $\mathcal{T}_{\xi}$ is a real analytic subvariety of $\mathcal{V}_{\xi}$.

It is easy to see that any complex Laurent polynomial $p\left(z_{1}, \ldots, z_{r}, z_{1}^{-1}, \ldots, z_{r}^{-1}\right)$, which vanishes on the torus $\left(S^{1}\right)^{r} \subset\left(\mathbf{C}^{*}\right)^{r}$, is identically zero. Hence, the intersection of any non-empty Zariski open subset of $\left(\mathbf{C}^{*}\right)^{r}$ with the torus $\mathcal{T}_{\xi}$ is non-empty.

This implies the following interpretation of the Novikov numbers:

3.3. Corollary. For a fixed $M$ and $\xi \in H^{1}(M ; \mathbf{R})$, consider the function

$$
T_{\xi} \ni L \mapsto \operatorname{dim}_{\mathbf{C}} H_{i}(M ; L) .
$$

There exists a proper real analytic subvariety $V=V_{i}(M) \subset \mathcal{T}_{\xi}$ such that for all $L \notin V$, the dimension $\operatorname{dim}_{\mathbf{C}} H_{i}(M ; L)$ equals to the Novikov number $b_{i}(\xi)$ and for $L^{\prime} \in V$

$$
\operatorname{dim}_{\mathbf{C}} H_{i}\left(M ; L^{\prime}\right)>b_{i}(\xi) .
$$

3.4. Proof of Theorem 5. Let $M$ be a closed manifold and let $\xi \in H^{1}(M ; \mathbf{R})$ be a real cohomology class. Consider the covering $\tilde{M} \rightarrow M$ corresponding to the subgroup $\pi^{\prime}=\operatorname{ker}(\xi) \subset \pi=\pi_{1}(M)$. Then $\pi / \pi^{\prime}$ is isomorphic to $\mathbf{Z}^{r}$ for some $r$.

Recall that the von Neumann Betti number $b_{i}^{(2)}\left(\tilde{M} ; \pi / \pi^{\prime}\right)$ is defined as follows. Fix a smooth triangulation of $M$ and consider the induced (equivariant) triangulation of the covering space $\tilde{M}$. Let $C_{*}(\tilde{M})$ denote the simplicial chain complex of this triangulation; it consists of free finitely generated $\mathbf{Z}\left[\mathbf{Z}^{r}\right]$-modules and $\mathbf{Z}\left[\mathbf{Z}^{r}\right]$-module homomorphisms. Consider the Hilbert space $L^{2}\left(\mathbf{Z}^{r}\right)$ with its canonical $\mathbf{Z}^{r}$-action and form the complex of Hilbert spaces

$$
L^{2}\left(\mathbf{Z}^{r}\right) \otimes_{\mathbf{Z}\left[\mathbf{Z}^{r}\right]} C_{*}(\tilde{M}) .
$$

The von Neumann Betti number $b_{i}^{(2)}\left(\tilde{M} ; \pi / \pi^{\prime}\right)$ is defined as the von Neumann dimension of the reduced homology of complex (3-5); cf. [A], [CG], [F2], and [L2].

Let us now recall the standard construction of harmonic analysis, providing an isomorphism between the space of $L^{2}$-functions on the torus $\left(S^{1}\right)^{r}$ with respect 
to the Lebesgue measure $d \mu$ (which will be assumed to be normalized so that the total torus $\left(S^{1}\right)^{r}$ has measure 1) and the space of $L^{2}$-functions on the lattice $\mathbf{Z}^{r}$. We will denote points of the lattice $\mathbf{Z}^{r}$ by $r$-tuples $\mathfrak{n}=\left(n_{1}, n_{2}, \ldots, n_{r}\right)$, where $n_{1}, \ldots, n_{r} \in \mathbf{Z}$. We will denote points of the torus $\left(S^{1}\right)^{r}$ by $z=\left(z_{1}, z_{2}, \ldots, z_{r}\right)$, where $z_{1}, \ldots, z_{r} \in S^{1} \subset \mathbf{C}$. The symbol $z^{\mathfrak{n}}$ will denote the product $z^{\mathfrak{n}}=z_{1}^{n_{1}} \cdot z_{2}^{n_{2}}$. $\cdots z_{r}^{n_{r}} \in S^{1}$. Any $L^{2}$-function $f \in L^{2}\left(\left(S^{1}\right)^{r}, d \mu\right)$ can be uniquely represented by its Fourier series

$$
f(z)=\sum_{\mathfrak{n} \in \mathbf{Z}^{r}} a_{\mathfrak{n}} z^{\mathfrak{n}} .
$$

The correspondence $f \mapsto\left(a_{\mathfrak{n}}\right)_{\mathfrak{n} \in \mathbf{Z}^{r}}$ defines an isometry $L^{2}\left(\left(S^{1}\right)^{r}, d \mu\right) \simeq L^{2}\left(\mathbf{Z}^{r}\right)$. It is important that under this isometry the multiplication by $z^{\mathfrak{m}}$ in $L^{2}\left(\left(S^{1}\right)^{r}, d \mu\right)$ (i.e. the map $\left.f(z) \mapsto z^{\mathfrak{m}} f(z)\right)$ transforms into the shift $\left(a_{\mathfrak{n}}\right)_{\mathfrak{n} \in \mathbf{Z}^{r}} \mapsto\left(a_{\mathfrak{n}}\right)_{\mathfrak{n}+\mathfrak{m} \in \mathbf{Z}^{r}}$ in $L^{2}\left(\mathbf{Z}^{r}\right)$; here $\mathfrak{m} \in \mathbf{Z}^{r}$.

The above identification allows us to view the Hilbert space $L^{2}\left(\mathbf{Z}^{r}\right)$ as the space of $L^{2}$-sections of a vector bundle over the torus $\mathcal{T}_{\xi}$, which we will now describe. Consider the real analytic line bundle $\mathcal{L} \rightarrow \mathcal{T}_{\xi}$ of unitary $\mathbf{Z}^{r}$-module structures on C. It is trivial as a vector bundle, and the fiber over a point $z \in \mathcal{T}_{\xi}$ has the following $\mathbf{Z}^{r}$-action: a point of the lattice $\mathfrak{n} \in \mathbf{Z}^{r}$ acts as multiplication by $z^{\mathfrak{n}} \in S^{1}$. We may form the chain complex

$$
\mathcal{L} \otimes \mathbf{z}^{r} C_{*}(\tilde{M})
$$

which is a real analytic family (parametrized by the points of the torus $\mathcal{T}_{\xi}$ ) of chain complexes of finite dimensional vector spaces. The $L^{2}$-complex (3-5) is isomorphic to the complex of $L^{2}$-sections of (3-6). Hence, applying Theorem 4.11 from [F3] we obtain that the von Neumann Betti number $b_{i}^{(2)}\left(\tilde{M} ; \pi / \pi^{\prime}\right)$ coincides with the generic Betti number $\operatorname{dim}_{\mathbf{C}} H_{i}(M ; L)$ for $L \in \mathcal{T}_{\xi}-V$, where $V$ is a proper real analytic subvariety. This may be also stated as the equality

$$
b_{i}^{(2)}\left(\tilde{M} ; \pi / \pi^{\prime}\right)=\int_{\mathcal{T}_{\xi}} \operatorname{dim}_{\mathbf{C}} H_{i}(M ; L) d \mu(L) .
$$

By Corollary 3.3 the generic Betti number is precisely the Novikov number $b_{i}(\xi)$. This completes the proof.

\section{REFERENCES}

[A] M. Atiyah, Elliptic operator, discrete groups and von Neumann algebras, Astérisque 32 (1976), 43 - 72. MR 54:8741

[Au] M. Audin, The Topology of Torus Action on Symplectic Manifolds, Birkäuser, 1991. MR 92m:57046

[BF1] M. Braverman, M. Farber, Novikov type inequalities for differential forms with non-isolated zeros, Math. Proc. of the Cambridge Phil. Society 122 (1997), 357 - 375. MR 99b:58220

[BF2] M.Braverman, M.Farber, Novikov-Bott inequalities., C.R.Acad. Sci. Paris 321 (1995), 895 - 902. MR 96i: 58165

[CG] J. Cheeger and M. Gromov, $L^{2}$-cohomology and group cohomology, Topology 25 (1986), 189 - 215. MR 87i:58161

[EG] Y. Eliashberg, M. Gromov, Lagrangian Intersection Theorey, Preprint (1996).

[F1] M. Farber, Exactness of the Novikov inequalities, Functional Analysis and its Applications 19:1 (1985), 40 - 49. MR 86g:58029

[F2] M. Farber, Homological algebra of Novikov - Shubin invariants and Morse inequalities., GAFA 6 (1996), 628 - 665. MR 97m:58034

[F3] M. Farber, Von Neumann categories and extended $L^{2}$ cohomology, Journal of K-theory (to appear). CMP 99:05 
[H] R. Hartshorne, Algebraic geometry, Springer-Verlag, 1977. MR 57:3116

[L1] W. Lück, $L^{2}$-Betti numbers of mapping tori and groups, Topology 33 (1994), 203 - 214. MR 95g:58235

[L2] W. Lück, $L^{2}$-invariants of regular coverings of compact manifolds and $C W$-complexes, To appear in "Handbook of Geometric Topology" (1999).

[MS] V. Mathai, M. Shubin, Twisted $L^{2}$ invariants of non-simply connected manifolds, Russian Journal of Math. Physics 4 (1996), 499 - 527. MR 98j:58112

[NS] S. Novikov and M. Shubin, Morse inequalities and von Neumann I $I_{1}$-factors., Soviet Math. Dokl. 34 (1987), 79 - 82. MR 88c:58065

[N] S.P. Novikov, The Hamiltonian formalism and a multivalued analogue of Morse theory, Russian Math. Surveys 37 (1982), 1-56. MR 84h:58032

School of Mathematical Sciences, Tel-Aviv University, Ramat-Aviv 69978, Israel

E-mail address: farber@math.tau.ac.il 